
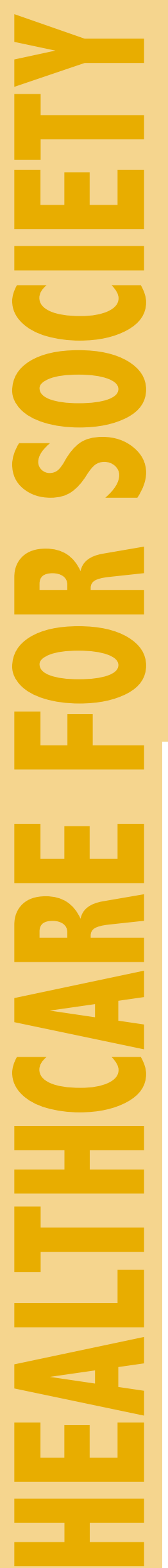

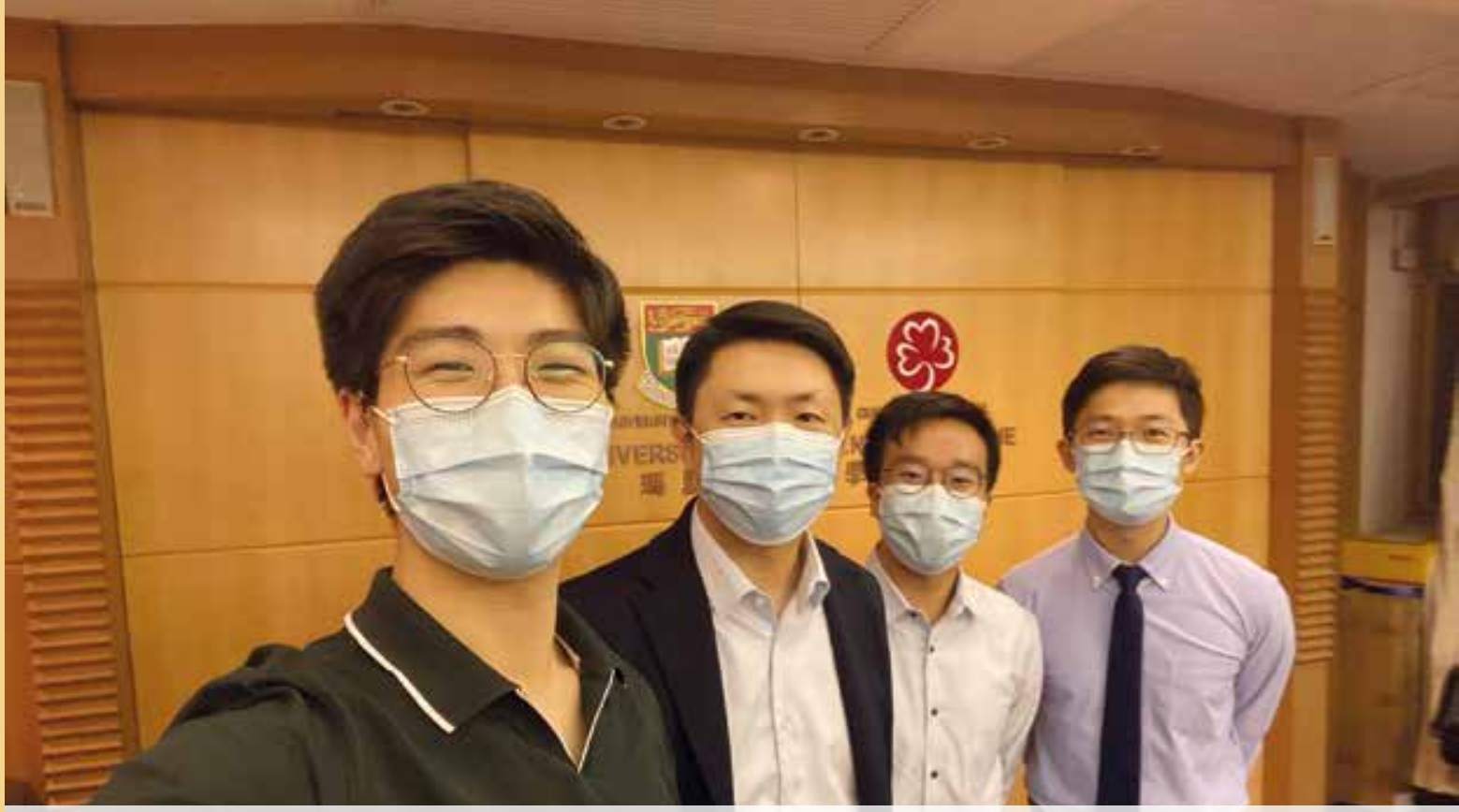

\title{
Growth in times of crisis: an interview with Professor Ivan Fan-ngai Hung
}

by: Gordon Chin' ${ }^{1}$, Justin Leung ${ }^{2}$, William Xue 1 Year 4 MB, ChB, The Chinese University of Hong Kong ${ }^{2}$ Year 5, MB, ChB, The Chinese University of Hong Kong ${ }^{3} \mathrm{MB}, \mathrm{ChB}$

https://doi.org/10.12809/hkmj-hc202112

Professor Ivan Fan-ngai Hung is currently in the public eye with his role in leading Hong Kong's response to the ongoing coronavirus 2019 (COVID-19) pandemic. As a co-convener of the Expert Committee on Clinical Events Assessment Following COVID-19 Immunisation under the Department of Health of the Hong Kong SAR Government, he is one of the public faces of the vaccination campaign. He is a Clinical Professor and Assistant Dean (Admissions) at the $\mathrm{Li} \mathrm{Ka}$ Shing Faculty of Medicine of The University of Hong Kong, as well as the Chief of the university's Infectious Diseases Division since 2018, specialising in infectious diseases as well as gastroenterology and hepatology. Prof Hung is also a Founding Member on the Board of the World Association for Infectious Diseases and Immunological Disorders.

Prof Hung did not envisage himself in such a position two decades ago. Since graduation from Bristol Medical School in 1996, Prof Hung had ambitions of becoming a gastroenterological surgeon. However, after returning to Hong Kong in 1999 and starting work at Queen Mary Hospital, he found that fate had other plans. By chance, he was offered the opportunity to join a new infectious diseases training position under the mentorship of Professor Kwok-yung Yuen, a keenly dedicated and hardworking doctor who still serves as a major influence and role model for Prof Hung. He accepted the offer just in time to be swept up in the severe acute respiratory syndrome (SARS) epidemic of 2003.

Prof Hung's experiences during the SARS epidemic remain etched into his mind. During the now infamous 'Amoy Gardens' outbreak at a residential complex in Kowloon, despite being only a trainee at the time, he joined a team of frontline medical officers. In the span of 3 days, these doctors truly went above and beyond their duties as they examined $>100$ patients with confirmed SARS at United Christian Hospital, collecting samples to investigate the identity of the pathogen. It was immensely stressful, as they were facing a completely unknown disease with high mortality, and with little available personal protective equipment at the time. Although the team feared for their safety, they maintained their faith and resolve. For Prof Hung, the incident was a turning point in his career, marking the beginning of his rise to prominence.

With the emergence of the new COVID-19 pandemic, it was Prof Hung's turn to take the lead. In contrast to the situation with SARS, hospitals in Hong Kong are now better equipped to handle such pathogens. Readily available isolation facilities, personal protective equipment, and infection control measures help protect doctors and other healthcare workers, and advancements in investigations and treatment options have improved things for patients. Similarly, the general public has learned 
important lessons from SARS, and now frequently follow better hand hygiene and facemask wearing practices to limit the spread of COVID-19. Indeed, everything might have changed after a decade, but Prof Hung's passion has remained the same. Whereas some people are becoming numb to the continuing COVID-19 facts and figures, Prof Hung still maintains the same degree of dedication and attention for each and every patient, even those very old or frail patients who stand a chance of surviving only with early treatment. "The key is the timing", he said. "We should never give up on any patients."

While Prof Hung takes great pride in his translational research-he has made several breakthroughs, such as demonstrating the treatment of severe swine flu with convalescent plasma and hyperimmune intravenous immunoglobulin, as well as the potentiation of an intradermal vaccine for influenza when used with a topical agent-public health education, particularly on COVID-19 early antiviral treatments and vaccinations especially for older adults, remains one of his top priorities. He took the initiative to hold a press conference every week at the beginning of the vaccination campaign, hoping to disseminate accurate information to the public and address any misunderstandings or misinformation. He also observed autopsies of patients who died after receiving a COVID-19vaccine in order to carefully determine the underlying cause. These acts, he believed, would give the public better confidence in the COVID-19 vaccines and maintain the momentum of the vaccination campaign.

In addition to his efforts to healthcare and education of Hong Kong citizens, Prof Hung contributed to the Hong Kong Anti-Cancer Society as an Infectious Diseases specialist. In this role, he advises immunocompromised patients with cancer to get vaccinations for various infectious diseases, including COVID-19, influenza and pneumococcal infection, and also to prevent herpes zoster reactivation as shingles. The Society, which aims to reduce the cancer burden in Hong Kong, makes various educational resources and videos on updated cancer therapies, cancer prevention, and infectious diseases prevention for patients with cancer (https:// www.hkacs.org.hk/en/).

The lessons Prof Hung would share with younger doctors and trainees are drawn from his personal experience. "Stand for your ambitions", he encourages us, emphasising how he was able to achieve his goal of being a gastroenterological specialist despite facing the major distraction of SARS early in his career. Nevertheless, he reminds us that the future is unpredictable, and that plans should not be set in stone: "Seize opportunities and be open to trying anything".

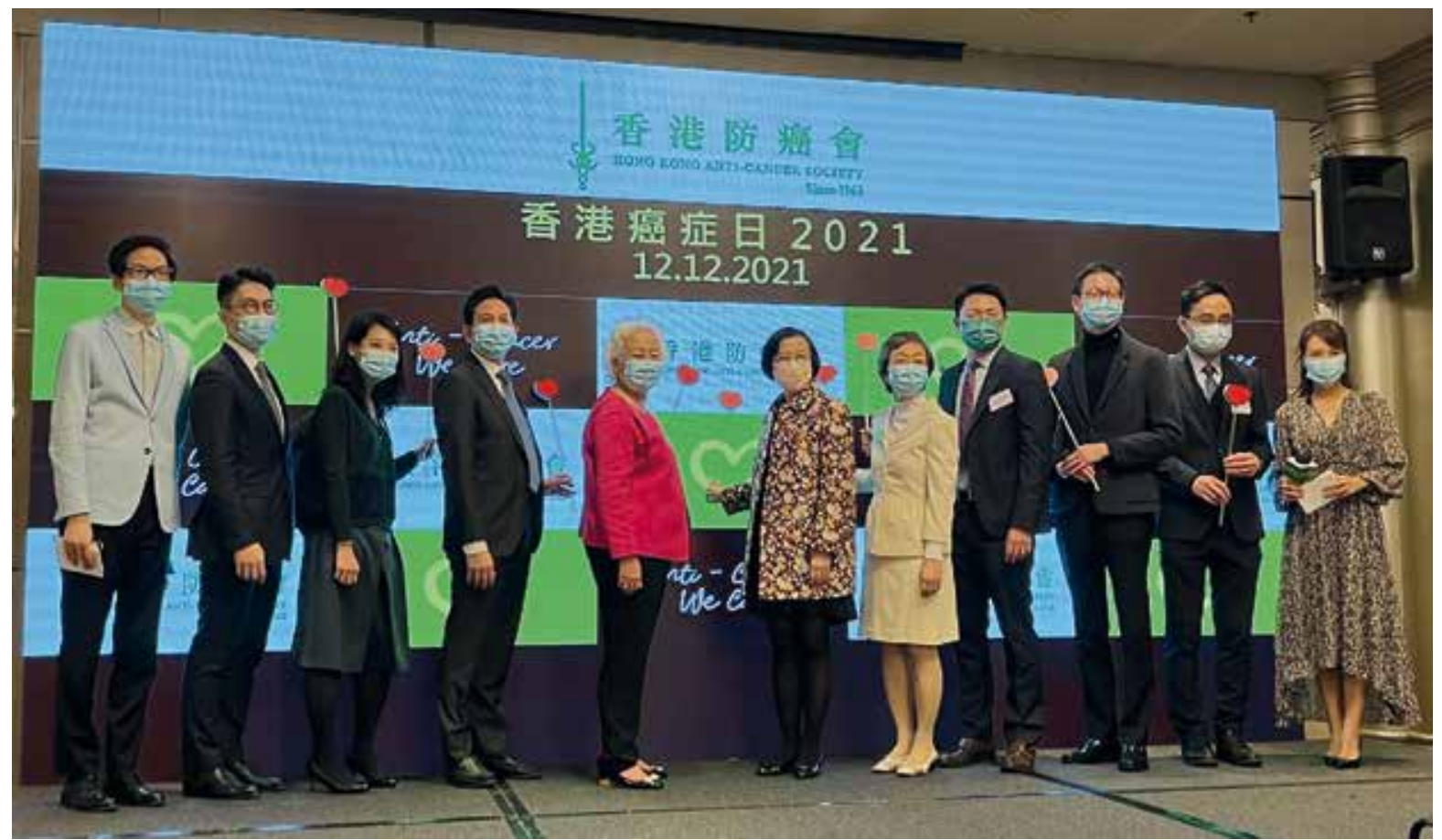

Prof Hung (8th from left) attending the launch of Hong Kong Anti-Cancer Day 2021, organised by the Hong Kong Anti-Cancer Society 